\title{
Peer Effects and Risk-Taking Among Entrepreneurs: Lab-in-the-Field Evidence
}

Maria Adelaida Lopera

Steeve Marchand

Mars / March 2017 


\section{Abstract}

We study how social interactions influence entrepreneurs' attitudes toward risk. We conduct two risk-taking experiments within workshops organized for young Ugandan entrepreneurs. Between the two experiments, the entrepreneurs participate in a networking activity where they build relationships and discuss with each other. We collect detailed data on peer network formation and on participants' choices before and after the networking activity. Our design implicitly controls for homophily effects (i.e. the tendency of individuals to develop relationships with people who have similar characteristics). We find that risk aversion is affected by social conformity. Participants tend to become more (less) risk averse in the second experiment if the peers they discuss with are on average more (less) risk averse in the first experiment. This suggests that social interactions play a role in shaping risk preferences.

Keywords: preference, risk aversion, entrepreneur, social norms JEL Classification: D03, D81, M13, Z13

Lopera : University of Munich (e-mail: malopera@econ.Imu.de)

Marchand : Université Laval (e-mail: steeve.marchand@ecn.ulaval.ca)

This study was carried out with financial and scientific support from the Partnership for Economic Policy (PEP) (www.pep-net.org) with funding from the Department for international Development (DFID) of the UK Aid and the government of Canada through the International Development Research Centre (IDRC). The authors are especially grateful to the team of PEP-researchers (PIERI-12451) lead by Juliet Ssekandi, who allowed us to join her evaluation project to collect experimental data. We also thank Benjamin Kachero and Samuel Galiwango for their extraordinary assistance in the field. We thank Charles Bellemare, Luc Bissonnette, Vincent Boucher and Bernard Fortin for useful comments that greatly improved the quality of this paper. We are grateful for the support provided by the PEP Research Director of Experimental Impact Evaluations Maria Laura Alzua, and by the PEP Scientific Advisor John Cockburn. This research benefited from collaborations with the Department for Children and Youth at the Ministry of Gender, Labor and Social Development (MGLSD), UNICEF-Uganda and Enterprise Uganda. Maria Lopera thanks the Fonds de recherche du Québec - Société et culture (FRQSC) for her scholarship, and Steeve Marchand thanks the Social Sciences and Humanities Research Council (SSHRC) for his scholarship. 


\section{Introduction}

Risk preferences play a fundamental role in economic decision-making. For instance, evidence suggests that entrepreneurship is associated with a higher than average tolerance toward risk (see Cramer et al., 2002, Ekelund et al., 2005 and Ahn, 2010). Conditional on entry, risk preferences may also affect businesses' success rates (Caliendo et al., 2010). But what factors contribute to an individual's risk tolerance? In this paper, we study the role of social interactions on risk preferences among groups of entrepreneurs. Using an original experimental design, we find a significant impact of conformism on risk-taking.

We conduct lab-in-the-field experiments on risk-taking within workshops organized for young entrepreneurs in Uganda. These workshops include a networking activity where entrepreneurs develop new relationships and converse with each other. We collect detailed information on who participants converse with during this activity. The entrepreneurs also participate in two risk-taking experiments: one before and one after the networking activity. These two experiments, combined with data on the peer network formation, allow us to evaluate whether social interactions have any effects on entrepreneurs' choices with respect to risk.

Our design allows us to bypass well-known empirical challenges in the estimation of peer effects, such as the reflection problem, first stated by Manski (1993). When the dependent variable and the peer variable are simultaneously determined, an individual's dependent variable is reflected in his peer variable via the social influence he exerts on his peers, which creates a simultaneity problem. This problem does not arise in our context because the peer variable is pre-determined. During the networking activity, participants can discuss choices made in the first experiment. Afterward, in the second experiment, participants make their choices individually, having possibly been influenced by the choices their peers made the first time. Thus, the dependent variable is determined in the second experiment while the peer variable is determined in the first, ruling out simultaneity problems.

Another empirical challenge in the estimation of peer effects is homophily: the tendency of individuals to develop relationships with people similar to themselves. Homophily creates a relationship between one's peer variable and his own choices even in the absence of peer effects, leading to identification issues. In other words, the peer network may be endogenous. There is a large and expanding literature that seeks to control for endogenous networks (for example, see Goldsmith-Pinkham and Imbens, 2013, Kelejian and Piras, 2014, Hsieh and Lee, 2016, Arduini et al., 2015, Qu and Lee, 2015 and Boucher, 2016). However, controlling for endogeneity necessarily requires strong assumptions. Our design allows us to identify peer effects in the presence of homophily under weaker assumptions. We use choices made in the two experiments to control 
for idiosyncratic individual characteristics through a first-difference approach. Assuming that individuals develop relationships based on these idiosyncratic characteristics is sufficient to rule out that the relationship between one's choice and those of her peers is caused by homophily. Furthermore, we can directly test for homophily effects. The choices made in our first experiment cannot possibly result from peer effects, because this experiment takes place before the networking activity. Therefore, the observed similarities between individuals' choices and those of the future peers they have not yet met can be used to identify homophily effects.

Our experiments are adaptations of the well-known Holt and Laury (2002) multiple choice lotteries designed to measure risk aversion. In these experiments, some combinations of choices are inconsistent with any given risk preference. While we find no evidence of homophily according to characteristics that affect our risk-aversion measure, we find that participants who make (in)consistent choices tend to develop relationships with individuals who also make (in)consistent choices. We also find significant social conformity effects: Participants tend to become more (less) risk averse in the second experiment if their peers were on average more (less) risk averse in the first experiment. This suggests that social interactions may contribute to shaping risk preferences. An entrepreneur could become more (less) risk averse following a relatively short discussion with a more (less) risk-averse entrepreneur. We expand our analysis by distinguishing between a preference to conform with peers who won (who made the choice that led to the highest payoff given the lotteries' results) from a preference to conform with those who lost (who made the choice that led to the lowest payoff given the lotteries' results). We find mixed evidence on how these effects differ, depending on whether participants face a set of choices that is directly comparable to the set previously presented to their peers. On the one hand, when this set of choices is identical, we find evidence of conformity with peers who won, but not with peers who lost. On the other hand, when the experiment is slightly different, we find that participants tend to conform with their peers regardless of the outcome.

While risk preferences are often assumed to be fixed, our findings suggest that they change in response to social interactions. We therefore contribute to the recent literature that suggests risk preferences vary across contexts (Barseghyan et al., 2011) and across time (Baucells and Villasís, 2010). ${ }^{1}$ Understanding the factors that drive these variations is of particular importance to understand decisions about becoming an entrepreneur. Evidence suggests that family dynamics are important in shaping individuals' preferences toward entrepreneurship. Dunn and Holtz-Eakin (2000) find that parental entrepreneurial experience is a

\footnotetext{
${ }^{1}$ Risk preferences may also be affected by emotional states such as joviality, sadness, fear and anger (Conte et al., forthcoming), or by stress (Cahlíková and Cingl, 2017).
} 
stronger predictor of entrepreneurship than an individual's or his parents' wealth. This correlation may result from both "nature" and "nurture" factors, but evidence suggests nurture factors play a significantly larger role (Lindquist et al., 2015). The social context outside of the family can also shape individuals' attitudes toward risk and entrepreneurship, or their beliefs or confidence about the expected returns of starting a business. It has been suggested, for instance, that having entrepreneurial peers can create non-monetary benefits of running a business (Giannetti and Simonov, 2009). Nanda and Sørensen (2010) find that individuals are more likely to become entrepreneurs if they work with peers who have previously been entrepreneurs. They argue that past workers' experience may spill over to their coworkers by influencing their entrepreneurial skills, knowledge or motivation. In this paper, we explore the complementary idea that entrepreneurs' risk preferences may also spill over to others.

Our paper more precisely contributes to the literature on peer effects on decisions made under risk, which has recently developed in other contexts. Bursztyn et al. (2014) study peer effects on the purchase of financial assets in a field experiment conducted at a financial brokerage. They find evidence of peer effects driven by both social learning (i.e. learning from peers) and social utility (i.e. utility that results directly from a peer's possession of an asset). Ahern et al. (2014) conduct an experiment about peer effects on risk aversion among MBA students and find significant peer effects. Gioia (2016) conducts a lab experiment and finds that the intensity of peer effects on risk-taking is determined in part by group identity: when peers are matched according to interest, the influence they exert on each other is greater. This suggests that peer effects might be important in our context, as our participants all share a common entrepreneurial identity.

Lahno and Serra-Garcia (2015) conduct a laboratory experiment to investigate whether participants' decisions about risk are influenced by their peers. They find that peer effects on risk-taking seem to be driven by a desire to conform with peers' choices. They argue that this implies that policymakers who seek to influence behaviours related to risk-taking (e.g. decisions to purchase insurance or acquire or repay debt) could publicly inform others about choices that are made by the population. This implication is particularly relevant for our paper, as we study real entrepreneurs. Our participants are people who need to finance their business projects with loans (this is discussed in detail in the next section). A policymaker could easily inform entrepreneurs about borrowing or insurance choices made by other entrepreneurs (for example, in an activity organized for them such as our workshops). He could also decide to make certain choices public in order to encourage specific behaviours (e.g. posting only the names of entrepreneurs who choose to insure their business). The policymaker could finally create networking activities aimed at discussing risk-taking decisions. These activities may generate social conformity effects that would push behaviours toward the 
average behaviour, reducing excessive risk-taking behaviours and increasing risk tolerance for excessively risk-averse individuals.

Our results also raise the issue of the direction of the causal relationship between risk preferences and the decision to start a business. If individuals who start a business enter a social world of entrepreneurs who tend to have higher risk tolerances, entry into entrepreneurship might cause more risk-taking. Cramer et al. (2002) raise the possibility of reverse causality, finding a negative effect of risk aversion on entry into entrepreneurship but questioning the causality of the relationship. Brachert and Hyll (2014) find that entry into entrepreneurship is associated with an increased willingness to take risks and argue that this entry may cause a change in risk attitudes for several reasons; our evidence suggests that social interactions with other entrepreneurs could be one of these reasons.

The next section describes our experimental design and data. Section 3 models participants' risk choices and presents the estimation of the social conformity effects. Section 4 concludes.

\section{Experimental Design and Data}

\subsection{The Workshops}

We contributed to the organization of six two-day workshops, along with the Partnership for Economic Policy ${ }^{2}$ a group of local researchers and UNICEF Uganda. The workshops took place in early 2014 in several locations in Uganda. ${ }^{3}$ Their primary aim was to evaluate and improve financial literacy among young Ugandan entrepreneurs. The workshops included training in finance and business planning, as well as a networking activity where entrepreneurs could share their knowledge with each other. Within each workshop, we ran two experiments on risk-taking: one before and one after the networking activity.

Entrepreneurs were recruited using U-report, a free Short Message Service (SMS) platform created by UNICEF to motivate and engage Ugandan youth into policymaking and governance. ${ }^{4}$ The first contact was an SMS message asking, "Are you an entrepreneur below 35 years old?" If the answer was affirmative, a second SMS message was sent: "Would you be interested in obtaining a credit loan from the Youth Venture Capital Fund?" This question aimed at selecting only entrepreneurs who were considering a business loan. If the answer was affirmative again, the potential participant received a phone call from a recruiter. The

\footnotetext{
${ }^{2}$ www.pep-net.org.

${ }^{3}$ Four workshops took place in the districts of Wakiso, M'bale, Gulu and M'barara. The other two workshops took place in the capital city of Kampala.

${ }^{4}$ Interested readers can visit www.unicef.org/uganda/voy.html for more information about the U-report platform.
} 
recruiter asked whether the potential participant was available for a two-day workshop near his/her home. Interested individuals were invited to the workshop, and the potential participant either accepted or rejected the invitation.

In total, 540 entrepreneurs participated in one of the workshops. Upon arrival, participants completed a survey about their sociodemographic characteristics. All subjects then participated in an initial risk-taking experiment, which we describe in the next subsection. After this experiment, subjects proceeded to the networking activity, which included a lunch and a discussion time. All participants in a given workshop were in the same room for both the lunch and the discussion time, which together lasted three to four hours. After the networking activity, we asked participants to write the names and identification numbers of up to seven participants with whom they had spent the most time chatting. They also had to identify each relationship as either an extended family member, a friend from before the workshop, or a person they met at the workshop. Once all participants had completed this questionnaire, a random sample of half the participants in each workshop (258 in total) was chosen to participate in a second risk-taking experiment, also described in the next subsection. ${ }^{5}$ The first day of the workshop then ended and participants returned home. The second day of the workshop included training in finance and business planning, which are outside the scope of this paper.

\section{$2.2 \quad$ The Risk-Taking Experiments}

All subjects participate in the first risk-taking experiment, which takes place before the networking activity. The experiment is an adapted version of the well-known Holt and Laury (2002) experiment designed to measure risk preferences. It consists of nine games in which participants must choose between two lotteries: a safe lottery or a risky lottery, with the risky lottery having more variability between the possible payoffs. Two large boxes are presented to participants, one representing each lottery. Each box is transparent and contains 40 large black and white balls, with the proportion of balls differing between the two boxes. The white balls represent low payoffs and black balls represent high payoffs. Participants also receive a paper questionnaire that provides them with the exact proportion of the two colours in each box. They are told that after all decisions are made, only one box will be selected at random, with one ball selected at random from inside that box. They will then be paid according to this ball's colour and the choice they made in the corresponding game. Decisions are made individually and participants are not allowed to consult each other. Appendix D provides additional details about how the experiment is presented to participants.

\footnotetext{
${ }^{5}$ Participants who were not selected for the second experiment received training in finance and business planning that was also part of the workshop, but which we do not address in this paper.
} 
Table 1 presents the two possible payoffs for each lottery. The amounts are substantial. For example, 10,000 Ugandan shillings (UGX), the highest possible payoff, represents more than 16 hours of work at Uganda's 2012-13 median wage. ${ }^{6}$

Each of the nine games has a different probability that the high payoff will be picked. This probability is shown in Table 2. It is low in the first game and increases for each game. The last column shows the difference in expected payoffs between choosing the safe lottery and choosing the risky lottery. The combination of choices made by an individual is informative of his preferences. For example, a risk-neutral individual should choose the safe lottery in games 1 to 5 , and then switch to the risky lottery in games 6 to 9 . Our main variable of interest - the risk-aversion measure - is the number of games in which the individual chooses the safe lottery. It ranges from 0 (all risky choices) to 9 (no risky choices).

Table 1: Game payoffs (in UGX)

\begin{tabular}{lcr}
\hline \hline & \multicolumn{2}{c}{ Return } \\
& Low & \multicolumn{1}{c}{ High } \\
\hline Safe lottery & 4,000 & 6,000 \\
Risky lottery & 1,000 & 10,000 \\
\hline
\end{tabular}

Table 2: Probability of high payoff in each game

\begin{tabular}{ccc}
\hline \hline Game & $\begin{array}{c}\text { Probability of } \\
\text { high payoff }\end{array}$ & $\begin{array}{c}\text { Expected payoff difference: } \\
\text { safe - risky (in UGX) }\end{array}$ \\
\hline 1 & $1 / 10$ & 4,100 \\
2 & $2 / 10$ & 3,200 \\
3 & $3 / 10$ & 2,300 \\
4 & $4 / 10$ & 1,400 \\
5 & $5 / 10$ & 500 \\
6 & $6 / 10$ & -400 \\
7 & $7 / 10$ & $-1,300$ \\
8 & $8 / 10$ & $-2,200$ \\
9 & $9 / 10$ & $-3,100$ \\
\hline
\end{tabular}

In theory, a participant should not switch his choice more than once. That is, if a participant chooses the safe lottery in game $k$ and the risky lottery in game $k+1$, it would be inconsistent to switch back to the safe lottery in game $k+2$. In practice, in our experiment as in other studies, some participants do switch more

\footnotetext{
${ }^{6}$ The median monthly earning in Uganda was about 110,000 UGX in 2012-13 for a paid employee, with the average work week comprised of approximately 41 hours. Because a month comprises 4.35 weeks on average, the average hourly earning is about 617 UGX per hour (see page 12 of the Uganda National Household Survey of 2012-13 [UBOS, 2014]).
} 
than once. ${ }^{7}$ This could be the result of a participant misunderstanding the experiment or having difficulty calculating the expected outcomes of each lottery. In the following sections, we will refer to a second outcome of interest: the consistency of choices, a dummy variable that equals one if the participant switches no more than once, and zero otherwise.

The participants in the second experiment (after the networking activity) are divided, within each workshop, into two subgroups. This creates 12 subgroups in total. Some subgroups replay the original experiment. The other subgroups play three different versions of the experiment, where we introduce an ambiguity component. For these groups, in the second experiment, a small proportion of the balls are wrapped in opaque bags so that participants cannot see whether they are black or white. The proportion of balls of unknown colour in the low, medium and high ambiguity groups are 5\%,10\% and 15\% respectively and remain fixed in all nine games. Participants are not provided any information about the distribution of the colours of the hidden balls. As for the balls that are not hidden, the proportions of white and black balls remain as described in Table 2. As we will see in Section 3, we will test whether there are any difference in peer effects when individuals face ambiguity. Appendix D provides details on all the experiments.

\subsection{Data}

Table 3 summarizes the data collected from the sociodemographic questionnaire, peer network questionnaire and the two risk-taking experiments' results. The average risk-aversion measure in the first experiment is 4.61 and slightly increases to 4.81 in the second experiment. The standard deviation of the differences in participants' risk-aversion measures in the two experiments is 1.80 . This indicates that the individual riskaversion measure varies upward and downward between the two experiments, even though the aggregate change is relatively small. The proportion of participants who make consistent choices in the first experiment is $54 \%$ and increases to $69 \%$ in the second second experiment. This increase could, among other things, be the result of playing the game a second time or of social learning effects.

On average, participants identify 4.54 peers who they met at the workshop and 1.76 peers who they knew before the workshop. Although we do not distinguish between these two types of peers in our main results, Appendix $\mathrm{C}$ shows that the significance of the peer effects we estimate in Section 3 mainly results from interactions between peers who have met at the workshop, ruling out the concern of social interactions that could have occurred before the networking activity.

\footnotetext{
${ }^{7}$ For example, see Holt and Laury (2002) and Jacobson et al. (2007).
} 
Table 3: Summary statistics

\begin{tabular}{|c|c|c|c|c|c|}
\hline & Mean & SD & Min. & Max. & Obs. \\
\hline \multicolumn{6}{|l|}{ Risk-aversion measure ( 0 to 9 ) } \\
\hline 1st experiment & 4.61 & 1.86 & 0 & 9 & 540 \\
\hline 2nd experiement & 4.83 & 1.91 & 0 & 9 & 258 \\
\hline Difference between 2 nd and 1st & 0.26 & 1.81 & -6 & 7 & 258 \\
\hline \multicolumn{6}{|l|}{ Consistency of choices ( 0 or 1$)$} \\
\hline 1st experiment & 0.54 & 0.50 & 0 & 1 & 540 \\
\hline 2nd experiment & 0.69 & 0.46 & 0 & 1 & 258 \\
\hline Difference between 2 nd and 1st & 0.16 & 0.56 & -1 & 1 & 258 \\
\hline \multicolumn{6}{|l|}{ Experiments' payoffs (in UGX) } \\
\hline 1st experiment & 5,025 & 3,193 & 1,000 & 10,000 & 540 \\
\hline 2nd experiment & 4,852 & 3,184 & 1,000 & 10,000 & 244 \\
\hline \multicolumn{6}{|l|}{ Number of peers } \\
\hline Met at the workshop & 4.52 & 2.35 & 0 & 7 & 540 \\
\hline Pre-existing & 1.76 & 2.15 & 0 & 7 & 540 \\
\hline Age & 26.63 & 4.41 & 17 & 50 & 540 \\
\hline Male & 0.82 & 0.38 & 0 & 1 & 540 \\
\hline \multicolumn{6}{|c|}{ Education (highest level completed) } \\
\hline Primary & 0.14 & 0.34 & 0 & 1 & 540 \\
\hline Secondary & 0.30 & 0.46 & 0 & 1 & 540 \\
\hline Technical & 0.30 & 0.46 & 0 & 1 & 540 \\
\hline University & 0.26 & 0.44 & 0 & 1 & 540 \\
\hline \multicolumn{6}{|l|}{ Workshop location } \\
\hline Kampala 1 & 0.17 & 0.37 & 0 & 1 & 540 \\
\hline Kampala 2 & 0.14 & 0.35 & 0 & 1 & 540 \\
\hline Wakiso & 0.17 & 0.37 & 0 & 1 & 540 \\
\hline M'bale & 0.19 & 0.39 & 0 & 1 & 540 \\
\hline Gulu & 0.19 & 0.39 & 0 & 1 & 540 \\
\hline M'barara & 0.15 & 0.35 & 0 & 1 & 540 \\
\hline \multicolumn{6}{|l|}{ Ambiguity level in 2nd exp. } \\
\hline None & 0.19 & 0.40 & 0 & 1 & 258 \\
\hline Low & 0.33 & 0.47 & 0 & 1 & 258 \\
\hline Medium & 0.30 & 0.46 & 0 & 1 & 258 \\
\hline High & 0.17 & 0.38 & 0 & 1 & 258 \\
\hline
\end{tabular}


After the second experiment, we asked participants to identify the main reason why they changed their choices between the two experiments (if they did change their choices). Table 4 presents the frequency of each possible answer among participants who reported having changed their choices. Almost $42 \%$ answered that the discussions they had with their peers during the networking activity had changed their mind. This suggests that participants discussed the experiment and choice strategies during the networking activity, even though we did not instruct them to. It also suggests that they influenced each others in these discussions.

Table 4: Self-reported reasons for changing choices in the 2nd experiment

\begin{tabular}{lcc}
\hline \hline Why did you change any of your choices? & Freq. & Percent \\
\hline I did not understand the first time & 18 & 12.08 \\
The game was different & 49 & 32.89 \\
Discussions with others changed my mind & 62 & 41.61 \\
I lost the first time & 20 & 13.42 \\
\hline Total & 149 & 100 \\
\hline \hline
\end{tabular}

\section{Social Interactions and Risk Preferences}

\subsection{The Empirical Models}

We model choices in the experiments as a trade-off an individual faces: choosing according to his own characteristics or according to his or her peers' choices. ${ }^{8}$ Let $y_{i r}$ be the risk-aversion measure of individual $i$ in experiment $r \in\{1,2\}$, where $r=1$ is the first experiment (before the networking activity) and $r=2$ is the second (after the networking activity). In the first experiment, individuals do not face the trade-off because they do not know their peers' choices. Participants simply choose $y_{i 1}$ according to their individual characteristics and maximize the following utility function:

$$
U_{i 1}\left(y_{i 1}\right)=-\frac{1}{2}\left(y_{i 1}-\alpha_{1}-\mathbf{x}_{i} \boldsymbol{\beta}-\eta_{i}-\epsilon_{i 1}\right)^{2}
$$

where $\mathbf{x}_{i}$ is a vector of individual $i$ 's observed characteristics and $\eta_{i}$ is the effect of his or her unobserved characteristics. Both $\mathbf{x}_{i}$ and $\eta_{i}$ are constant over time (i.e. $\forall r \in\{1,2\}$ ). These characteristics may include

\footnotetext{
${ }^{8}$ See Bisin et al. (2006), Boucher (2016) and Boucher and Fortin (2016) for other examples that model this trade-off in a similar way.
} 
the individual's idiosyncratic risk preferences. Thus, we allow for these preferences to be specific to the individual and to be a function of individual characteristics. This is consistent with the literature, which finds differences in risk preferences across individuals (for example, see Croson and Gneezy (2009), who find gender-based differences in risk preferences).

The error term $\epsilon_{i 1}$ is specific to $i$ and to the first experiment. It allows for shocks, such as stress or other emotions, which might temporally affect preferences (see Cahlíková and Cingl (2017) and Conte et al. (forthcoming). The error term also acknowledges that we do not directly observe risk preferences, but rather an imperfect measure of it. ${ }^{9}$ Thus, in the spirit of Baucells and Villasís (2010), our risk-aversion measure could be the result of both risk preferences and a random error component.

The first-order condition is:

$$
y_{i 1}=\alpha_{1}+\mathbf{x}_{i} \boldsymbol{\beta}+\eta_{i}+\epsilon_{i 1} .
$$

In the second experiment $(r=2)$ after the networking activity, participants face a trade-off between staying true to their own characteristics and conforming with their peers' choices. We model social conformity using two specifications: homogeneous peer effects, where participants partly conform with the average behaviour of their peers, and heterogeneous peer effects, where participants may conform differently with different peers according to the first experiment's results.

\subsubsection{Homogeneous peer effects specification}

We assume that individual $i$ in the second experiment maximizes the following utility function:

$$
U_{i 2}\left(y_{i 2}\right)=-\frac{1}{2}\left(y_{i 2}-\alpha_{1}-\alpha_{2}-\alpha_{2}^{g}-\mathbf{x}_{i} \boldsymbol{\beta}-\delta W_{i}-\eta_{i}-\epsilon_{i 2}\right)^{2}-\frac{\theta}{2}\left(y_{i 2}-\frac{1}{n_{i}} \sum_{j \in N_{i}} y_{j 1}\right)^{2}
$$

where $n_{i}$ is $i$ 's number of peers and $N_{i}$ is his set of peers. The first part on the right-hand side is the private component of the utility function and the second is its social component. Utility is decreasing with the distance between the individual's choice and the average choice of his peers. We allow for the possibility that playing the game a second time affects risk aversion in some way through the parameter $\alpha_{2}$. We also include $\alpha_{2}^{g}$, a dummy variable specific to the ambiguity-level fixed effect $g \in\{$ none, low, medium, high $\}$ (recall from

\footnotetext{
${ }^{9}$ Preference elicitation methods other than Holt and Laury (2002) lotteries could lead to different measures (Anderson and Mellor, 2009).
} 
the last section that participants in the second experiment are randomly assigned to games with different ambiguity levels). We thus allow for each of these four games to have a different effect on the utility that results from choices. We set the reference category to $g=$ none so that $\alpha_{2}^{\text {none }}=0 . W_{i}$ is the individual's payoff from the first experiment (divided by 1,000 ), so that $\delta$ may capture wealth effects. ${ }^{10}$ The parameter $\theta$ is the social conformity effect, modelled as a preference to conform with peers' average behaviour. We allow this parameter to differ depending on whether the participant faces ambiguity or not, so that we have:

$$
\theta= \begin{cases}\theta_{n a} & \text { if } g=\text { none } \\ \theta_{a} & \text { otherwise }\end{cases}
$$

Therefore, $\theta_{n a}$ is the social conformity effect of participants who participate in the exact same experiment the second time, whereas $\theta_{a}$ is the social conformity effect for those who participate in one of the games that includes ambiguity. ${ }^{11}$ Substituting equation (2) into equation (3) yields:

$$
U_{i 2}\left(y_{i 2}\right)=-\frac{1}{2}\left(y_{i 2}-y_{i 1}-\alpha_{2}-\alpha_{2}^{g}-\delta W_{i}-\epsilon_{i}\right)^{2}-\frac{\theta}{2}\left(y_{i 2}-\frac{1}{n_{i}} \sum_{j \in N_{i}} y_{j 1}\right)^{2}
$$

where $\epsilon_{i} \equiv \epsilon_{i 2}-\epsilon_{i 1}$. Importantly, this first-difference approach in the private component of the utility function writes off $\mathbf{x}_{i} \boldsymbol{\beta}$ and $\eta_{i}$. The first-order condition is: ${ }^{12}$

$$
y_{i 2}=\frac{1}{1+\theta}\left(\alpha_{2}+\alpha_{2}^{g}+y_{i 1}+\delta W_{i}+\frac{\theta}{n_{i}} \sum_{j \in N_{i}} y_{j 1}+\epsilon_{i}\right) .
$$

Equation (6) provides an empirical model we can estimate. The model allows us to bypass two major empirical challenges in the estimation of peer effects. First, because the peer variable $\left(\frac{1}{n_{i}} \sum_{j \in N_{i}} y_{j 1}\right)$ is predetermined, the simultaneity problem described by Manski (1993) does not arise. Second, the model implicitly controls for homophily (i.e. the tendency individuals have to develop relationships with people similar to themselves). Homophily is usually a concern in the estimation of peer effects. Individuals may match according to observable variables (e.g. gender, age, education), which is generally not a problem because these

\footnotetext{
${ }^{10}$ The results we will present are robust to using the logarithm of the payoff instead, or to not controlling for the payoff. ${ }^{11}$ Separate peer effect estimates for all levels of ambiguity (low, medium, high) are available upon request.

${ }^{12}$ If the individual has no peers $\left(n_{i}=0\right)$, the utility function simplifies to $U_{i 2}\left(y_{i 2}\right)=-\frac{1}{2}\left(y_{i 2}-y_{i 1}-\alpha_{2}-\alpha_{2}^{g}-\delta W_{i}-\epsilon_{i}\right)^{2}$ and the first-order condition becomes $y_{i 2}=\alpha_{2}+\alpha_{2}^{g}+y_{i 1}+\delta W_{i}+\epsilon_{i}$. Only one individual in our sample did not report having any peers. As we will see below, we estimate the model using nonlinear least squares, which allows to estimate this individual's first-order condition jointly with those of other individuals. Furthermore, all the results we present are robust to removing this individual.
} 
variables' effects can be controlled for. A more important concern is the possibility of homophily according to unobserved characteristics that might affect the variable of interest. In our model, this would mean that individuals with similar values of $\eta_{i}$ would tend to become peers. This would imply a correlation between $y_{i r}$ and the average outcome of i's peers even in the absence of peer effects. Fortunately, our first-difference approach in the private component of the utility function cancels out $\eta_{i}$ in equation (5), so the remaining error term $\epsilon_{i}$ is independent of peers' average outcome.

\subsubsection{Heterogeneous peer effects specification}

We now allow for heterogeneous peer effects between peers who won and peers who lost in the first experiment. We define "winning" ("loosing") as having made the choice that led to the highest (lowest) payoff given the game and the ball that were picked at random in the first experiment. Let $N_{i}^{w}$ be the set of peers of $i$ who

won in the first experiment and $N_{i}^{l}$ be the set of peers who lost. Additionally, let $n_{i}^{w}$ and $n_{i}^{l}$ be the respective numbers of $i$ 's peers in these two groups (so $n_{i}=n_{i}^{w}+n_{i}^{l}$ ). Our model with heterogeneous peer effects becomes:

$$
\begin{aligned}
U_{i 2}\left(y_{i 2}\right)= & -\frac{1}{2}\left(y_{i 2}-y_{i 1}-\alpha_{2}-\alpha_{2}^{g}-\delta W_{i}-\epsilon_{i}\right)^{2} \\
& -\frac{\theta^{w} n_{i}^{w}}{2 n_{i}}\left(y_{i 2}-\frac{1}{n_{i}^{w}} \sum_{j \in N_{i}^{w}} y_{j 1}\right)^{2}-\frac{\theta^{l} n_{i}^{l}}{2 n_{i}}\left(y_{i 2}-\frac{1}{n_{i}^{l}} \sum_{j \in N_{i}^{l}} y_{j 1}\right)^{2},
\end{aligned}
$$

where $\theta^{k}$ is the social conformity effect for the peer group $k \in\{w, l\}$, modelled as a preference to conform with this group's average behaviour. The relative importance of each group is weighted by the proportion of peers in each category $n_{i}^{k} / n_{i}$. The first-order condition is:

$$
y_{i 2}=\frac{n_{i}}{n_{i}+\theta^{w} n_{i}^{w}+\theta^{l} n_{i}^{l}}\left(\alpha_{2}+\alpha_{2}^{g}+y_{i 1}+\delta W_{i}+\frac{\theta^{w}}{n_{i}} \sum_{j \in N_{i}^{w}} y_{j 1}+\frac{\theta^{l}}{n_{i}} \sum_{j \in N_{i}^{l}} y_{j 1}+\epsilon_{i}\right),
$$

which we use as an empirical model for estimation.

\subsection{Estimation and Results}

We estimate our two specifications (equations 6 and 8) using nonlinear least squares (NLS). NLS relies on the assumption that the expected value of the error term, conditional on explanatory and predetermined 
variables, is zero. Thus, it relies on weaker assumptions than other nonlinear methods, such as maximum likelihood estimation, that rely on distributional assumptions. ${ }^{13}$ Table 5 presents the results for our two specifications. We use the sandwich estimator of variance to calculate standard errors. Column (a) shows the estimates for the homogeneous peer effects specification. The peer effect $\theta_{n a}$ (for those who participated in the same experiment the second time) is 0.783 and is significant at the 10 percent level. Thus, the estimated marginal effect (the effect of a one unit increase in peers' average risk-aversion measure) is $\hat{\theta}_{n a} /\left(1+\hat{\theta}_{n a}\right)=$ 0.439. For the participants who participated in a treatment with ambiguity the second time, we find a lower social conformity effect $\left(\hat{\theta}_{a}=0.627\right)$. This effect is more precisely estimated and significant, possibly because of the higher number of participants who participated in a treatment with ambiguity.

Column (b) presents the results of the heterogeneous specification. For those who participated in the same experiment (without ambiguity) the second time, we find that participants tend to conform with their peers who won the first time. Conversely, we find a negative but not statistically significant conformity effect from peers who lost. Furthermore, we reject the null hypothesis that social conformity effects from peers who won and those from peers who lost are equal. On the contrary, for participants who played a different game (with ambiguity) in the second experiment, we find positive social conformity effects from the two peer groups and do not reject that the two are equal. This suggests that peer effects can arise differently depending on whether or not the choices available are directly comparable to the observed peer choices. When this is not the case, individuals may simply conform with their peers' choices regardless of the outcome. Overall, our findings suggest a significant impact of conformism on risk-taking decisions. We also find that having won a higher payoff in the first experiment tends to make individuals more willing to take risks.

As mentioned previously, our model implicitly controls for homophily because of the first difference approach in the private component of the utility function. Nevertheless, we test for the presence of homophily in Appendix B. Homophily according to observable characteristics can be tested for by looking at whether individuals tend to be peers with others who share these observable characteristics. Furthermore, because we observe behaviours before social interactions occur, we can also test for homophily on unobservable characteristics that affect the outcome. We do so by testing for correlations in outcomes between future peers who have not yet met. This correlation cannot possibly come from peer effects and should therefore be attributable to homophily.

Appendix B provides no evidence of homophily according to observable or unobservable characteristics that affect our measure of risk aversion. However, Appendix B shows that participants who make (in)consistent

\footnotetext{
${ }^{13}$ See chapter 5 of Cameron and Trivedi (2005) for explanations on nonlinear estimators.
} 
Table 5: Peer effects on the risk-aversion measure Nonlinear least squares estimation

\begin{tabular}{|c|c|c|}
\hline & $\begin{array}{l}\text { Hom. } \\
\text { effects } \\
\text { (a) }\end{array}$ & $\begin{array}{l}\text { Het. } \\
\text { effects } \\
\text { (b) }\end{array}$ \\
\hline $\begin{array}{l}\text { Peer effect - } \\
\text { no ambiguity } \theta_{n a}\end{array}$ & $\begin{array}{l}0.783^{*} \\
(0.459)\end{array}$ & \\
\hline $\begin{array}{l}\text { Peer effect - } \\
\text { ambiguity } \theta_{a}\end{array}$ & $\begin{array}{l}0.627^{* * *} \\
(0.184)\end{array}$ & \\
\hline $\begin{array}{l}\text { Peer effect from winners - } \\
\text { no ambiguity } \theta_{n a}^{w}\end{array}$ & & $\begin{array}{l}1.207^{* *} \\
(0.594)\end{array}$ \\
\hline $\begin{array}{l}\text { Peer effect from losers - } \\
\text { no ambiguity } \theta_{n a}^{l}\end{array}$ & & $\begin{array}{l}-0.935 \\
(0.734)\end{array}$ \\
\hline $\begin{array}{l}\text { Peer effect from winners - } \\
\text { ambiguity } \theta_{a}^{w}\end{array}$ & & $\begin{array}{l}0.387^{* *} \\
(0.164)\end{array}$ \\
\hline $\begin{array}{l}\text { Peer effect from losers - } \\
\text { ambiguity } \theta_{a}^{l}\end{array}$ & & $\begin{array}{l}1.261^{* *} \\
(0.496)\end{array}$ \\
\hline Second exp. effect $\alpha_{2}$ & $\begin{array}{l}1.122^{*} \\
(0.594)\end{array}$ & $\begin{array}{l}1.439^{* *} \\
(0.602)\end{array}$ \\
\hline $\begin{array}{l}\text { 1st exp. payoff effect } \delta \\
\text { (in thousands of UGX) }\end{array}$ & $\begin{array}{l}-0.200^{* * *} \\
(0.069)\end{array}$ & $\begin{array}{l}-0.223^{* * *} \\
(0.073)\end{array}$ \\
\hline $\begin{array}{l}p \text {-value } H_{0}: \theta_{n a}^{w}=\theta_{n a}^{l} \\
p \text {-value } H_{0}: \theta_{a}^{w}=\theta_{a}^{l}\end{array}$ & & $\begin{array}{l}0.05 \\
0.09\end{array}$ \\
\hline Number of observations & 258 & 258 \\
\hline Ambiguity fixed effects $\alpha_{2}^{g}$ & Yes & Yes \\
\hline
\end{tabular}


choices tend to develop relationships with participants who also make (in)consistent choices. This suggests the presence of homophily based on cognitive skills. Finally, Appendix A shows that, after controlling for this homophily, there is no evidence of social influence effects on the consistency of choices. That is, we find no social learning effects from discussions with peers who made consistent choices.

\section{Conclusion}

In this paper, we combine information on the formation of a network of entrepreneurs with observations from a field experiment on choices under risk before and after social interactions occur. This design allows us to estimate social conformity effects while controlling for homophily. We find that entrepreneurs tend to conform with their peers' choices, which suggests that social interactions play a role in shaping risk preferences. Our results suggest that a policymaker could influence these entrepreneurs' risk-related choices, such as decisions about loans or insurance, by making other entrepreneurs' choices public. He could also influence risk-taking behaviours by organizing networking activities aimed at discussing risk-taking decisions. Social conformity effects may push behaviours toward the average behaviour, reducing excessive risk-taking behaviours and increasing risk tolerance for excessively risk-averse individuals.

The social interactions captured in our experiment are authentic; we do not influence the network formation or the discussions participants have. Furthermore, the peer effects we estimate result from a three- to fourhour-long networking activity. Our finding that these few hours of free discussion time are enough to influence one's choices raises the question of whether more durable interactions would have an even greater effect. When people develop long-lasting social relationships, long-lasting peer effects may contribute to shaping individuals' risk attitudes in the long run. Dohmen et al. (2012) find evidence that long-run risk attitudes are shaped during childhood through attitudes transmitted by parents and local environment. Our findings provide evidence that the transmission of risk attitudes may continue into adulthood.

\section{References}

Ahern, K. R., R. Duchin, And T. Shumway (2014): "Peer effects in risk aversion and trust," Review of Financial Studies, 27, 3213-3240.

Ahn, T. (2010): "Attitudes toward risk and self-employment of young workers," Labour Economics, 17, $434-442$. 
Anderson, L. R. And J. M. Mellor (2009): "Are risk preferences stable? Comparing an experimental measure with a validated survey-based measure," Journal of Risk and Uncertainty, 39, 137-160.

Arduini, T., E. Patacchini, E. Rainone, et Al. (2015): "Parametric and Semiparametric IV Estimation of Network Models with Selectivity," Tech. rep., Einaudi Institute for Economics and Finance (EIEF).

Barseghyan, L., J. Prince, And J. C. Teitelbaum (2011): "Are risk preferences stable across contexts? Evidence from insurance data," The American Economic Review, 101, 591-631.

BAucells, M. AND A. Villasís (2010): "Stability of risk preferences and the reflection effect of prospect theory," Theory and Decision, 68, 193-211.

Bisin, A., U. Horst, AND O. ÖzGür (2006): "Rational expectations equilibria of economies with local interactions," Journal of Economic Theory, 127, 74-116.

Boucher, V. (2016): "Conformism and self-selection in social networks," Journal of Public Economics, 136, $30-44$.

Boucher, V. And B. Fortin (2016): "Some Challenges in the Empirics of the Effects of Networks," in The Oxford Handbook of the Economics of Networks, ed. by Y. Bramoullé, A. Galeotti, and B. Rogers, chap. 12.

Brachert, M. ANd W. Hyll (2014): "On the stability of preferences: Repercussions of entrepreneurship on risk attitudes," SOEPpapers on Multidisciplinary Panel Data Research.

Bramoullé, Y. And B. Fortin (2010): "Social networks: econometrics," The New Palgrave Dictionary of Economics.

Brock, W. A. And S. N. Durlauf (2001): "Discrete choice with social interactions," The Review of Economic Studies, 68, 235-260.

Bursztyn, L., F. Ederer, B. Ferman, and N. Yuchtman (2014): "Understanding mechanisms underlying peer effects: Evidence from a field experiment on financial decisions," Econometrica, 82, 1273-1301.

Cahlíková, J. And L. Cingl (2017): "Risk preferences under acute stress," Experimental Economics, 20, 209-236.

Caliendo, M., F. Fossen, And A. Kritikos (2010): "The impact of risk attitudes on entrepreneurial survival," Journal of Economic Behavior \&3 Organization, 76, 45-63. 
Cameron, A. C. And D. L. Miller (2015): “A practitioner's guide to cluster-robust inference," Journal of Human Resources, 50, 317-372.

Cameron, A. C. And P. K. Trivedi (2005): Microeconometrics: methods and applications, Cambridge University Press.

Chandrasekhar, A. (2016): "Econometrics of Network Formation," in The Oxford Handbook of the Economics of Networks, ed. by Y. Bramoullé, A. Galeotti, and B. Rogers, chap. 13.

Conte, A., M. V. Levati, And C. NARdi (forthcoming): "Risk preferences and the role of emotions," Economica.

Cramer, J. S., J. Hartog, N. Jonker, and C. M. Van PraAg (2002): "Low risk aversion encourages the choice for entrepreneurship: An empirical test of a truism," Journal of economic behavior $\mathcal{G}$ organization, $48,29-36$.

Croson, R. And U. Gneezy (2009): "Gender differences in preferences," Journal of Economic literature, $47,448-474$.

Dohmen, T., A. Falk, D. Huffman, and U. Sunde (2012): "The intergenerational transmission of risk and trust attitudes," The Review of Economic Studies, 79, 645-677.

Dunn, T. And D. Holtz-Eakin (2000): "Financial capital, human capital, and the transition to selfemployment: Evidence from intergenerational links," Journal of Labor Economics, 18, 282-305.

Ekelund, J., E. Johansson, M.-R. Järvelin, and D. Lichtermann (2005): "Self-employment and risk aversion-evidence from psychological test data," Labour Economics, 12, 649-659.

Giannetti, M. And A. Simonov (2009): "Social interactions and entrepreneurial activity," Journal of Economics \&3 Management Strategy, 18, 665-709.

GioiA, F. (2016): "Peer effects on risk behaviour: the importance of group identity," Experimental Economics, 1-30.

Goldsmith-Pinkham, P. and G. W. Imbens (2013): "Social networks and the identification of peer effects," Journal of Business \&3 Economic Statistics, 31, 253-264. 
Holt, C. A. And S. K. Laury (2002): "Risk aversion and incentive effects," American Economic Review, $92,1644-1655$.

Hsien, C.-S. AND L. F. LeE (2016): "A social interactions model with endogenous friendship formation and selectivity," Journal of Applied Econometrics, 31, 301-319.

Jacobson, S., R. Petrie, et Al. (2007): "Inconsistent Choices in Lottery Experiments: Evidence from Rwanda," Journal of Risk and Uncertainty.

Kelejian, H. H. And G. Piras (2014): "Estimation of spatial models with endogenous weighting matrices, and an application to a demand model for cigarettes," Regional Science and Urban Economics, 46, 140-149.

Lahno, A. M. And M. Serra-Garcia (2015): "Peer effects in risk taking: Envy or conformity?" Journal of Risk and Uncertainty, 50, 73-95.

Lindquist, M. J., J. Sol, And M. Van PraAg (2015): "Why do entrepreneurial parents have entrepreneurial children?" Journal of Labor Economics, 33, 269-296.

Manski, C. F. (1993): "Identification of endogenous social effects: The reflection problem," The review of economic studies, $60,531-542$.

Nanda, R. And J. B. Sørensen (2010): "Workplace peers and entrepreneurship," Management Science, $56,1116-1126$.

QU, X. AND L.-F. LEE (2015): "Estimating a spatial autoregressive model with an endogenous spatial weight matrix," Journal of Econometrics, 184, 209-232.

Uganda Bureau of Statistics [UBOS] (2014): "Uganda National Household Survey 2012/2013." Tech. rep. 


\section{Appendix}

\section{A Social Interactions and Consistency of Choices}

\section{A.1 The Empirical Models}

In this section, we investigate the effects of social interactions on another outcome: the consistency of choices. We assume participants make some effort to understand how to make good choices. This implies a different model underlying participants' choices than the one described in Section 3. Let the latent variable $e_{i r}^{*}$ be the effort that an individual $i$ puts in understanding experiment $r \in\{1,2\}$. Assume participants have to reach some minimal level of understanding, normalized to 0, to make consistent choices. This leads to the standard latent variable framework:

$$
e_{i r}= \begin{cases}1 & \text { if } \quad e_{i r}^{*} \geq 0 \\ 0 & \text { otherwise }\end{cases}
$$

where $e_{i r}$ is the consistency of choices that results from putting enough effort into understanding the experiment. Assume participants choose a level of effort to maximize their utility. In the first experiment $(r=1)$, they choose the effort that maximizes the following utility:

$$
V_{i 1}\left(e_{i 1}^{*}\right)=\left(c_{1}+\mathbf{x}_{i} \gamma+\mu_{i}+\psi_{i 1}\right) e_{i 1}^{*}-\frac{e_{i 1}^{*}}{2}
$$

where $\mathbf{x}_{i}$ and $\mu_{i}$ are the individual's fixed observed and unobserved characteristics, respectively, and $\psi_{i 1}$ is an

error term. The first portion of the right-hand side represents the individual's perceived benefit from exerting effort, while the second portion represents the increasing cost of effort. The perceived benefit of effort depends on individual characteristics. For example, a low-skill person (low $\mathbf{x}_{i}$ or $\mu_{i}$ ) may not see why he should try to calculate anything, and instead prefer to pick lotteries at random. Conversely, individual characteristics could be seen as affecting the cost of effort: a high-skill person may find it less costly to provide sufficient effort to understand the experiment. The first-order condition is:

$$
e_{i 1}^{*}=c_{1}+\mathbf{x}_{i} \gamma+\mu_{i}+\psi_{i 1}
$$

After the networking activity, a random subgroup participates in the second experiment and may now be 
influenced by the discussion they had with their peers. Let $m_{i}$ be the number of $i$ 's peers who made consistent choices in the first experiment. Assume that, for the second experiment, individual $i$ chooses effort $e_{i 2}^{*}$ in order to maximize:

$$
V_{i 2}\left(e_{i 2}^{*}\right)=\left(c_{1}+c_{2}+c_{2}^{g}+\mathbf{x}_{i} \gamma+\mu_{i}+\epsilon_{i 2}\right) e_{i 2}^{*}-\frac{e_{i 2}^{*}}{2}+\lambda m_{i} e_{i 2}^{*}
$$

where $c_{2}$ is a constant that adds to the first experiment's constant. It might (among other things) capture a learning effect of doing the experiment a second time or a fatigue effect. We again add ambiguity dummies $c_{2}^{g}$ specific to the level of ambiguity $g \in\{$ none, low, medium, high $\}$ in the second experiment. The reference category is set to $g=$ none so that $c_{2}^{\text {none }}=0$. The individual's perceived utility is affected by his peers through social learning effects. The $m_{i}$ peers who understood the experiment the first time may make it easier for $i$ to understand the experiment because he can learn from them. We can see this as a reduction in the cost of effort needed to understand the experiment. As in the last section, we let the peer effect $\lambda$ differ for those who participated in a treatment that included ambiguity the second time, so that:

$$
\lambda= \begin{cases}\lambda_{n a} & \text { if } g=\text { none } \\ \lambda_{a} & \text { otherwise }\end{cases}
$$

The first-order condition is:

$$
e_{i 2}^{*}=c_{1}+c_{2}+c_{2}^{g}+\mathbf{x}_{i} \gamma+\lambda m_{i}+\mu_{i}+\epsilon_{i 2}
$$

which provides an empirical model we can estimate. Once again, the peer variable $m_{i}$ is predetermined, which rules out the reflection problem of Manski (1993). It also rules out the multiple equilibriums problem that arises in binary outcome models where the dependent variable and the peer variables are simultaneously determined (Brock and Durlauf, 2001).

\section{A.1.1 Naive Specification}

Contrary to the last section, the latent variable framework implies we cannot use the first-difference approach to remove equation (14)'s constant observed or unobserved variables. Thus, if there is homophily according to $\mu_{i}, m_{i}$ should be correlated with the error term. Nevertheless, as a benchmark, we first ignore homophily concerns and use equation (14) as our empirical model assuming $E\left(\mu_{i}+\epsilon_{i} \mid m_{i}, \mathbf{x}_{i}\right)=0$. 


\section{A.1.2 Difference-in-Differences Specification}

Homophily and peer effects may both create similarities in peers' choices in the second experiment. However, in the first experiment, only homophily can create these similarities. We can therefore use the choices in the first experiment to separately identify the two effects.

We use a specification analogous to a difference-in-differences (DID) estimation. In a standard DID setting, a control group and a treatment group are observed both before and after a treatment occurs. The variation in the outcome of interest that occurs between the two periods for reasons other than the treatment can be controlled for using the variation in this outcome among the control group. The additional variation that is specific to the treatment group is then attributed to the treatment effect.

In our setting, the number of peers who made consistent choices $\left(m_{i}\right)$ is analogous to the DID treatment variable. As in a standard DID estimation, individuals with different values of of $m_{i}$ may on average have different levels of understanding about the experiment, even before social interactions occur, because of homophily. The variation in the outcome that occurs between our two experiments for reasons other than social interactions can also be controlled for using a dummy variable that equals 1 if $r=2$ and 0 otherwise. The additional variation that arises in the the second experiment as a function of $m_{i}$ can then be used to identify peer effects. Specifically, we estimate the following model:

$$
e_{i r}^{*}=c_{1}+\mathbf{x}_{i} \gamma+\tilde{\lambda} m_{i}+\mathbb{1}(r=2)\left[c_{2}+c_{2}^{g}+\lambda m_{i}\right]+\mu_{i}+\epsilon_{i r}
$$

where $\mathbb{1}(r=2)$ equals 1 if $r=2$ and 0 otherwise. The covariance between $m_{i}$ and $\mu_{i}$ that comes from homophily is present in the two experiments and is thus captured by $\tilde{\lambda}$. Besides homophily effects, the

estimate of $\tilde{\lambda}$ captures any relationship between $\mu_{i}$ and $m_{i}$ that arises for reasons other than the social interactions occurring after the first experiment. Thus, $\lambda$ excludes the effect of homophily and captures the peer effects, which only arise in the second experiment.

\section{A.2 Estimation and Results}

We estimate our two specifications using probit estimations. Table 6 presents the estimated average marginal effects. We include in $\mathbf{x}_{i}$ age, sex and education, as well as fixed effects for the six locations in which the experiments took place. Column (a) presents the naive specification (equation 14) and column (b) presents the DID specification (equation 15). The number of observations in column (b) is greater because we use the choices from the first experiment to control for homophily. The standard errors are clustered by individual, 
but the results are robust to using the sandwich estimator of variance without clustering. ${ }^{14}$

The naive peer effect estimates show a significant relationship between an individual's consistency of choices and his number of peers who made consistent choices in the first experiment. However, this relationship is significant only for participants who participated in an experiment without ambiguity the second time. The relationship may, however, include both a peer effects and a homophily effect.

Our DID estimation yields a significant homophily effect. An individual's probability of making consistent choices in the first experiment is 3.9 percentage points greater, on average, for each peer who made consistent choices, even if participants have not yet discussed with each other. The additional effect of the number of peers who made consistent choices in the second experiment - the social learning effect of having met and discussed with these peers - is not significant. Therefore, we can see that neglecting the role of homophily would have led us to interpret the relationship between one's consistency of choices and those of her peers as peer effects.

To complement and test the robustness of our results, we test for the presence of homophily using a simple network formation model in Appendix B. We again find evidence of homophily according to unobserved characteristics that affects the consistency of choices, $e_{i 1}$. Specifically, we find that individual $i$ has a higher probability of becoming peers with individual $j$ if $\left|e_{i 1}-e_{j 1}\right|$ equals 0 than if it equals 1 , after controlling for individual variables and for similarities in $i$ and $j$ 's variables. Because $e_{i 1}$ and $e_{j 1}$ are determined before social interactions occur, this effect is not caused by peer effects and can thus be attributed to homophily.

\section{B Testing for homophily}

Let the network tie $d_{i j}$ be equal to 1 if individual $i$ states that individual $j$ is his new friend and 0 otherwise. We allow the network to be directed, meaning that $d_{i j}$ is not necessarily equal to $d_{j i}$. As suggested by Bramoullé and Fortin (2010), we let the probability that $d_{i j}=1$ depend on the absolute distance between $i$ and $j$ 's variables (which capture homophily effects) and on both $i$ and $j$ 's variables. We model individual $i$ 's decision to state that $j$ is one of his friends by the following rule:

$$
d_{i j}^{*}=\delta_{0}+\mathbf{x}_{i} \boldsymbol{\delta}_{1}+\mathbf{x}_{j} \boldsymbol{\delta}_{2}+y_{i 1} \delta_{3}+y_{j 1} \delta_{4}+\left|\mathbf{x}_{i}-\mathbf{x}_{j}\right| \boldsymbol{\rho}_{x}+\left|y_{i 1}-y_{j 1}\right| \rho_{y}+v_{i j}
$$

\footnotetext{
${ }^{14}$ We avoid clustering by the six locations (on top of the locations' fixed effects), because clustering with too few clusters leads to a downward-biased variance matrix estimate, and thus to over-rejection. However, small cluster sizes may also lead to a biased estimate of the variance matrix. See Cameron and Miller (2015) for a discussion on problems that arise with few clusters or with small clusters.
} 
Table 6: Peer effects on consistency of choices Average marginal effects of a probit estimation

\begin{tabular}{|c|c|c|}
\hline & $\begin{array}{l}\text { Naive } \\
\text { (a) }\end{array}$ & $\begin{array}{l}\text { DID } \\
\text { (b) }\end{array}$ \\
\hline $\begin{array}{l}\text { Peer effect - } \\
\text { no ambiguity } \lambda_{n a}\end{array}$ & $\begin{array}{l}0.113^{* *} \\
(0.049)\end{array}$ & $\begin{array}{c}0.044 \\
(0.049)\end{array}$ \\
\hline $\begin{array}{l}\text { Peer effect - } \\
\text { ambiguity } \lambda_{a}\end{array}$ & $\begin{array}{c}0.025 \\
(0.023)\end{array}$ & $\begin{array}{l}-0.020 \\
(0.026)\end{array}$ \\
\hline Homophily effect $\tilde{\lambda}$ & & $\begin{array}{l}0.039^{* * *} \\
(0.014)\end{array}$ \\
\hline 2nd exp. effect $c_{2}$ & & $\begin{array}{c}0.011 \\
(0.204)\end{array}$ \\
\hline \multicolumn{3}{|c|}{ Observable characteristics } \\
\hline Age & $\begin{array}{l}-0.001 \\
(0.006)\end{array}$ & $\begin{array}{l}-0.004 \\
(0.004)\end{array}$ \\
\hline Male & $\begin{array}{l}0.066 \\
(0.076)\end{array}$ & $\begin{array}{l}0.149^{* * *} \\
(0.048)\end{array}$ \\
\hline Education: secondary & $\begin{array}{l}0.337^{* * *} \\
(0.104)\end{array}$ & $\begin{array}{l}0.173^{* * *} \\
(0.062)\end{array}$ \\
\hline Education: technical & $\begin{array}{l}0.233^{* *} \\
(0.110)\end{array}$ & $\begin{array}{l}0.170^{* * *} \\
(0.061)\end{array}$ \\
\hline Education: university & $\begin{array}{l}0.324^{* * *} \\
(0.106)\end{array}$ & $\begin{array}{l}0.233^{* * *} \\
(0.062)\end{array}$ \\
\hline Number of observations & 258 & 798 \\
\hline Number of individuals & 258 & 258 \\
\hline $\begin{array}{l}\text { Ambiguity fixed effects } c_{2}^{g} \\
\text { District fixed effects }\end{array}$ & $\begin{array}{l}\text { Yes } \\
\text { Yes }\end{array}$ & $\begin{array}{l}\text { Yes } \\
\text { Yes }\end{array}$ \\
\hline
\end{tabular}




$$
d_{i j}= \begin{cases}1 & \text { if } \quad d_{i j}^{*}>0 \\ 0 & \text { otherwise }\end{cases}
$$

We call $\boldsymbol{\rho}_{x}$ the vector of homophily according to observable characteristics effects and $\rho_{y}$ the effect of homophily on unobservable characteristics (that affect $\left.y_{i 1}\right)$. Importantly, the outcome variables $\left(y_{i r}\right.$ and $\left.y_{j r}\right)$ are those of the first experiment $(r=1)$ before social interactions occur, so that $\rho_{y}$ may not capture peer effects.

We estimate this model using a probit estimation. Because this is a model of peer network formation, we remove observations where peers stated that they already knew each other before the workshop. It is important to note that this model has many weaknesses in explaining some features of the network formation. It assumes that the probability that $i$ and $j$ become peers is independent of other links formed in the network. Thus, this model may not explain clustering (i.e. the stylized fact that two individuals who share a peer in common have a higher probability of becoming peers with each other). One should consult Chandrasekhar (2016) for a review of econometric models that are more consistent with stylized facts. Nevertheless, this simple model allows us to test for the existence of homophily effects. We also estimate the above model for our other variable of interest - the consistency of choices - by redefining $y_{i 1}$ and $y_{j 1}$ by binary variables that equal 1 if individuals $i$ and $j$, respectively, made consistent choices in the first experiement and 0 otherwise. Finally, we estimate a model that includes both variables.

The three specifications are presented in Table 7. We find no evidence of homophily according to observable variables. We also do not find evidence of homophily according to unobserved characteristics that affect the risk-aversion measure. However, we do find significant homophily effects according to unobserved characteristics that affect the consistency of choices.

\section{Peer effects estimates from pre-existing vs. new peers}

As mentioned in Section 2.3, most of the social relationships we observe were developed at workshops between individuals who did not know each other previously. Participants have on average 4.54 peers they met at the workshop and 1.76 peers they knew from before the workshop. Table 8 presents separate peer effect estimates from these two types of peers. Column (a) is the homogeneous peer is the homogeneous peer effects specification - exactly the same as column (a) from our main results presented in Table 5. Column (b) shows heterogeneous peer effects from "pre-existing" and "new" peers, where "pre-existing" peers refer to those the 
Table 7: Average marginal effects of a probit estimate - dependent variable: friendship

\begin{tabular}{llll}
\hline \hline & $(\mathrm{a})$ & $(\mathrm{b})$ & $(\mathrm{c})$ \\
\hline
\end{tabular}

Absolute value of the difference

between individual variables

Consistency of choices

Risk-aversion measure

Age
Male

Education

$\begin{array}{rrrr}-0.0046 & * * & & -0.0046 \\ (0.0020) & & & (0.0020) \\ & & -0.0000 & -0.0001 \\ & (0.0006) & (0.0006) \\ -0.0003 & -0.0003 & -0.0003 \\ (0.0003) & (0.0003) & (0.0003) \\ 0.0000 & 0.0000 & 0.0000 \\ (0.0000) & (0.0000) & (0.0000) \\ -0.0000 & -0.0000 & -0.0000 \\ (0.0006) & (0.0006) & (0.0006)\end{array}$

Individual's variable

Consistency of choices

0.0021

$(0.0020)$

Risk-aversion measure

Age
Male
Education
0.0002
$(0.0002)$

0.0000

$(0.0000)$

$-0.0000$

$(0.0006)$

0.0008
$(0.0005)$
0.0002
$(0.0002)$
0.0000
$(0.0000)$
0.0000
$(0.0006)$

0.0022

$(0.0021)$

0.0008

$(0.0005)$

0.0002

$(0.0002)$

0.0000

$(0.0000)$

$-0.0000$

$(0.0006)$

\section{Potential peer's variable}

\begin{tabular}{|c|c|c|c|}
\hline Consistency of choices & $\begin{array}{r}-0.0020 \\
(0.0020)\end{array}$ & & $\begin{array}{r}-0.0020 \\
(0.0021)\end{array}$ \\
\hline Risk-aversion measure & & $\begin{array}{r}-0.0005 \\
(0.0006)\end{array}$ & $\begin{array}{r}-0.0005 \\
(0.0006)\end{array}$ \\
\hline \multirow[t]{2}{*}{ Age } & 0.0002 & 0.0002 & 0.0002 \\
\hline & $(0.0002)$ & $(0.0002)$ & $(0.0002)$ \\
\hline \multirow[t]{2}{*}{ Male } & 0.0000 & 0.0000 & 0.0000 \\
\hline & $(0.0000)$ & $(0.0000)$ & $(0.0000)$ \\
\hline \multirow[t]{2}{*}{ Education } & -0.0000 & -0.0000 & -0.0000 \\
\hline & $(0.0006)$ & $(0.0006)$ & $(0.0006)$ \\
\hline Number of obs. & 47,664 & 47,664 & 47,664 \\
\hline $\begin{array}{l}\text { Notes: } \\
1 \text { - Dummy variables for the } \\
\text { took place are also included in } \\
2 \text { - Standard errors are cluster } \\
* * * p \leq 0.01 ;{ }^{* *} p \leq 0.05 ;{ }^{*} p\end{array}$ & $\begin{array}{l}\text { which th } \\
\text { ession bu } \\
\text { vo poten }\end{array}$ & $\begin{array}{l}\text { iment } \\
\text { ot shown. } \\
\text { cs" identif }\end{array}$ & \\
\hline
\end{tabular}


individual already knew before the workshop and "new" peers refers to those met at the workshop. The empirical model is the same as equation (8), except that "winner" and "loser" types of peers are replaced by "pre-existing" and "new" types of peers. The results show that the significance of our peer effect estimates is mostly driven by the interactions that occurred for the first time at the workshops.

\section{Details about the experiments}

Upon arrival to the workshop, participants answered a questionnaire about their socio-demographic characteristics. They were then gathered in a room for the first experiment. An instructor explained the instructions and verified participants' comprehension by asking a series of questions. When he thought everyone understood, he took the box representing the first lottery and put it in front of the group. The box contained black balls (representing a high payoff) and white balls (representing a low payoff). He briefly explained again the composition of the box and asked participants to write down their first investment choice on a decision sheet. Figures 1 and 2 show the decision sheets. The first lottery corresponds to box 1.1. The box indicates the exact proportion of each ball and their associated payoffs. When participants were done writing their choice, the instructor took the box representing the second lottery and briefly explained the composition of the box, before participants recorded their second choice of lottery. Then the instructor went on with the third lottery and onward. All choices were made individually and in silence. Once everyone had finished recording their choices, one of the nine lotteries was randomly chosen by drawing from a bag of balls numbered from 1 through 9. Then, a single ball was randomly drawn from the selected lottery and participants were payed according to the choice recorded on their decision sheet.

Approximately $50 \%$ of participants were then randomly chosen to participate in a second experiment. Selected participants were randomly divided into two groups, with each group participating in an experiment with a different level of ambiguity (including none, low, medium and high). Only two ambiguity treatments were conducted at each workshop. Table 9 shows the number of participants assigned to each ambiguity level at each workshop. Note that there are more participants assigned to the low and medium levels. This comes from a confusion that arose in the organization of one of the workshops. Specifically, the participants of the "Kampala 2" workshop should have been assigned with none and high levels of ambiguity, but were mistakenly assigned with low and medium instead. This, however, does not invalidate our results, as we control for these differences in ambiguity levels in our estimations.

Participants assigned to none participated in the same experiment as the first experiment. Those assigned 
Table 8: Peer effects on the risk-aversion measure heterogeneous effects between pre-existing and new peers -

Nonlinear least squares estimation

\begin{tabular}{|c|c|c|}
\hline & $\begin{array}{l}\text { Hom. } \\
\text { effects } \\
\text { (a) }\end{array}$ & $\begin{array}{l}\text { Het. } \\
\text { effects } \\
\text { (b) }\end{array}$ \\
\hline $\begin{array}{l}\text { peer effect - } \\
\text { no ambiguity } \theta_{n a}\end{array}$ & $\begin{array}{c}0.783^{*} \\
(0.459)\end{array}$ & \\
\hline $\begin{array}{l}\text { peer effect - } \\
\text { ambiguity } \theta_{a}\end{array}$ & $\begin{array}{l}0.627^{* * *} \\
(0.184)\end{array}$ & \\
\hline $\begin{array}{l}\text { peer effect from pre-existing peers - } \\
\text { no ambiguity } \theta_{n a}^{p}\end{array}$ & & $\begin{array}{l}-0.109 \\
(0.317)\end{array}$ \\
\hline $\begin{array}{l}\text { peer effect from new peers - } \\
\text { no ambiguity } \theta_{n a}^{n}\end{array}$ & & $\begin{array}{c}2.047^{*} \\
(1.118)\end{array}$ \\
\hline $\begin{array}{l}\text { peer effect from pre-existing peers - } \\
\text { ambiguity } \theta_{a}^{p}\end{array}$ & & $\begin{array}{c}0.905^{*} \\
(0.497)\end{array}$ \\
\hline $\begin{array}{l}\text { peer effect from new peers - } \\
\text { ambiguity } \theta_{a}^{n}\end{array}$ & & $\begin{array}{l}0.575^{* * *} \\
(0.200)\end{array}$ \\
\hline second exp. effect $\alpha_{2}$ & $\begin{array}{l}1.122^{*} \\
(0.594)\end{array}$ & $\begin{array}{l}1.565^{* * *} \\
(0.509)\end{array}$ \\
\hline $\begin{array}{l}\text { 1st exp payoff effect } \delta \\
\text { (in thousands of UGX) }\end{array}$ & $\begin{array}{l}-0.200^{* * *} \\
(0.069)\end{array}$ & $\begin{array}{l}-0.206^{* * *} \\
(0.064)\end{array}$ \\
\hline $\begin{array}{l}p \text {-value } H_{0}: \theta_{n a}^{w}=\theta_{n a}^{l} \\
p \text {-value } H_{0}: \theta_{a}^{w}=\theta_{a}^{l}\end{array}$ & & $\begin{array}{l}0.08 \\
0.54\end{array}$ \\
\hline number of observations & 258 & 258 \\
\hline Ambiguity fixed effects $\alpha_{2}^{g}$ & Yes & Yes \\
\hline
\end{tabular}


to treatments with ambiguity were presented a box that contained, in addition to white and black balls, balls that were wrapped in opaque bags, so that their colour was unknown. The decisions sheets for the low, medium and high ambiguity treatments are presented in figures 3 to 8 .

Figure 1: Decision sheet for the first experiment

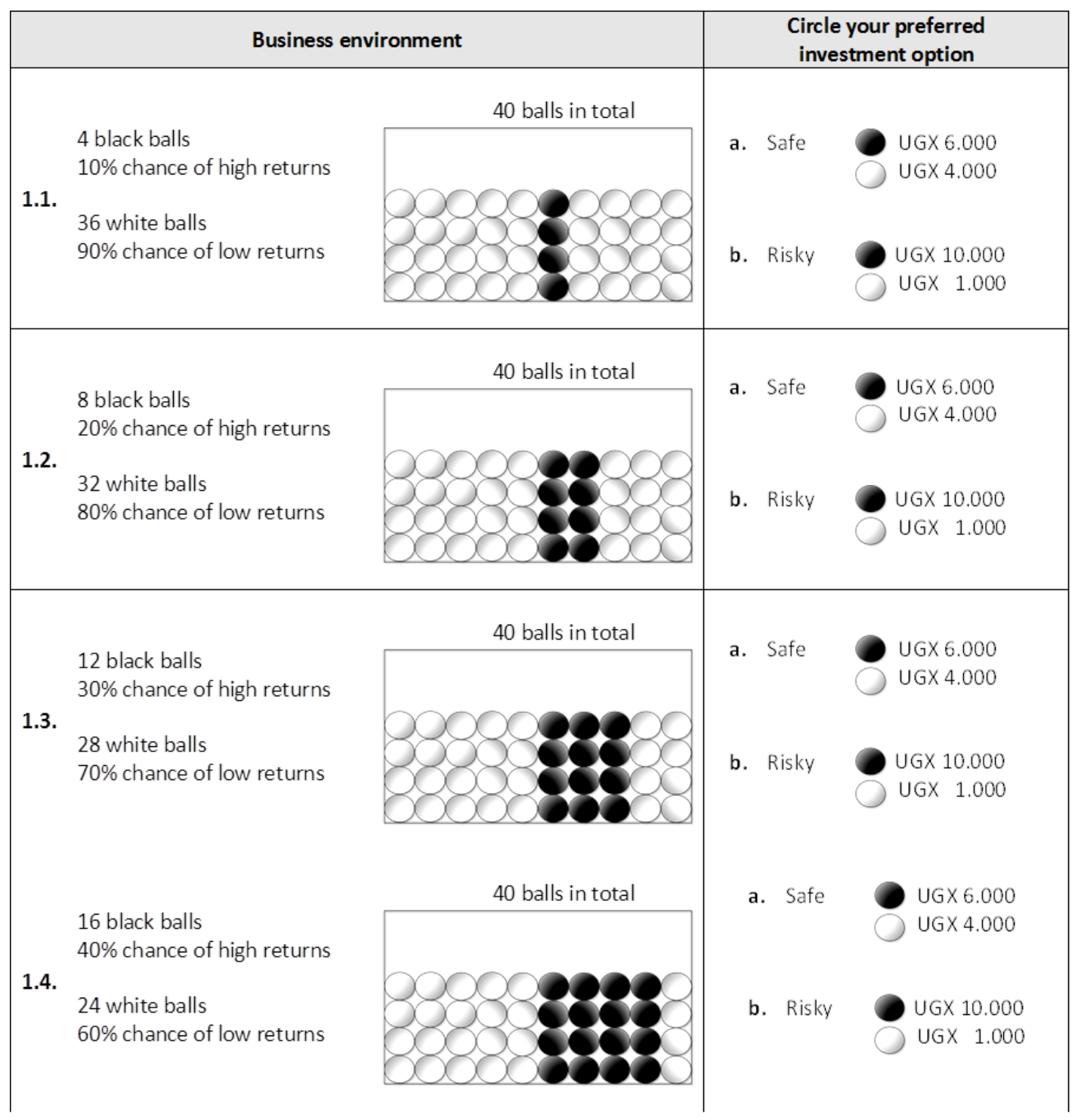


Table 9: Assignment of participants to the second experiment

\begin{tabular}{|c|c|c|c|c|c|c|c|}
\hline \multirow[b]{2}{*}{ District } & & \multirow[b]{2}{*}{ 1st exp. only } & \multicolumn{4}{|c|}{ Ambiguity level in second experiment } & \multirow[b]{2}{*}{ Tota } \\
\hline & & & None & Low & Medium & High & \\
\hline Kampala 1 & $\begin{array}{c}\text { Obs. } \\
\%\end{array}$ & $\begin{array}{c}53 \\
59 \%\end{array}$ & $\begin{array}{c}0 \\
0 \%\end{array}$ & $\begin{array}{c}0 \\
0 \%\end{array}$ & $\begin{array}{c}18 \\
20 \%\end{array}$ & $\begin{array}{c}19 \\
21 \%\end{array}$ & $\begin{array}{c}90 \\
100 \%\end{array}$ \\
\hline Kampala 2 & $\begin{array}{c}\text { Obs. } \\
\%\end{array}$ & $\begin{array}{c}44 \\
57 \%\end{array}$ & $\begin{array}{c}0 \\
0 \%\end{array}$ & $\begin{array}{c}18 \\
23 \%\end{array}$ & $\begin{array}{c}15 \\
19 \%\end{array}$ & $\begin{array}{c}0 \\
0 \%\end{array}$ & $\begin{array}{c}77 \\
100 \%\end{array}$ \\
\hline Wakiso & $\begin{array}{c}\text { Obs. } \\
\%\end{array}$ & $\begin{array}{c}46 \\
51 \%\end{array}$ & $\begin{array}{c}0 \\
0 \%\end{array}$ & $\begin{array}{c}24 \\
26 \%\end{array}$ & $\begin{array}{c}21 \\
23 \%\end{array}$ & $\begin{array}{c}0 \\
0 \%\end{array}$ & $\begin{array}{c}91 \\
100 \%\end{array}$ \\
\hline M'bale & $\begin{array}{c}\text { Obs. } \\
\%\end{array}$ & $\begin{array}{c}50 \\
50 \%\end{array}$ & $\begin{array}{c}24 \\
24 \%\end{array}$ & $\begin{array}{c}0 \\
0 \%\end{array}$ & $\begin{array}{c}0 \\
0 \%\end{array}$ & $\begin{array}{c}26 \\
26 \%\end{array}$ & $\begin{array}{c}100 \\
100 \%\end{array}$ \\
\hline Gulu & $\begin{array}{c}\text { Obs. } \\
\%\end{array}$ & $\begin{array}{c}50 \\
49 \%\end{array}$ & $\begin{array}{c}26 \\
25 \%\end{array}$ & $\begin{array}{c}27 \\
26 \%\end{array}$ & $\begin{array}{c}0 \\
0 \%\end{array}$ & $\begin{array}{c}0 \\
0 \%\end{array}$ & $\begin{array}{c}103 \\
100 \%\end{array}$ \\
\hline M'barara & $\begin{array}{c}\text { Obs. } \\
\%\end{array}$ & $\begin{array}{c}39 \\
49 \%\end{array}$ & $\begin{array}{c}0 \\
0 \%\end{array}$ & $\begin{array}{c}16 \\
20 \%\end{array}$ & $\begin{array}{c}24 \\
30 \%\end{array}$ & $\begin{array}{c}0 \\
0 \%\end{array}$ & $\begin{array}{c}79 \\
100 \%\end{array}$ \\
\hline Total & $\begin{array}{c}\text { Obs. } \\
\%\end{array}$ & $\begin{array}{c}282 \\
52 \%\end{array}$ & $\begin{array}{c}50 \\
9 \%\end{array}$ & $\begin{array}{c}85 \\
16 \%\end{array}$ & $\begin{array}{c}78 \\
14 \%\end{array}$ & $\begin{array}{l}45 \\
8 \%\end{array}$ & $\begin{array}{c}540 \\
100 \%\end{array}$ \\
\hline
\end{tabular}


Figure 2: Decision sheet for the first experiment (cont.)

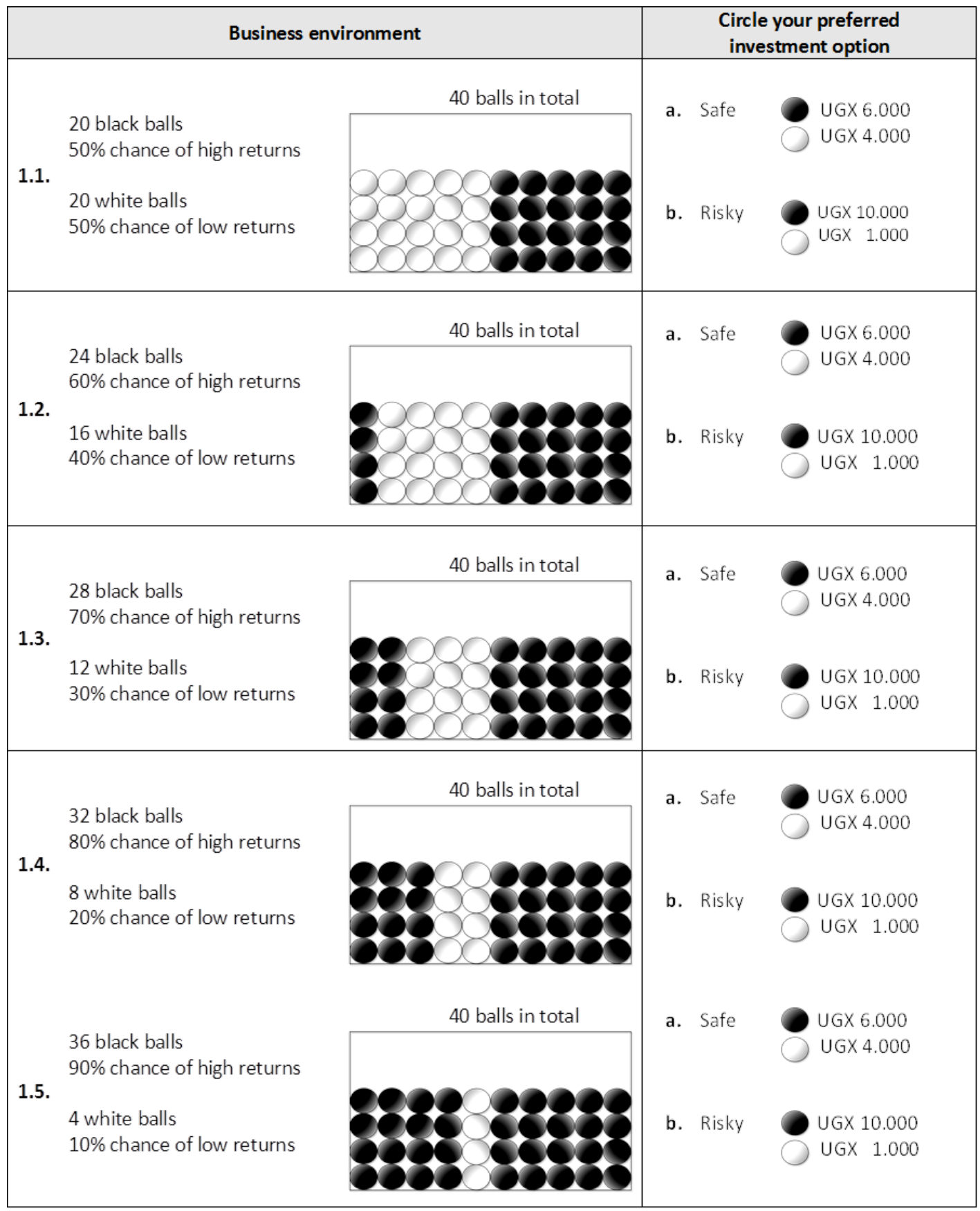


Figure 3: Decision sheet for the second experiment with low ambiguity

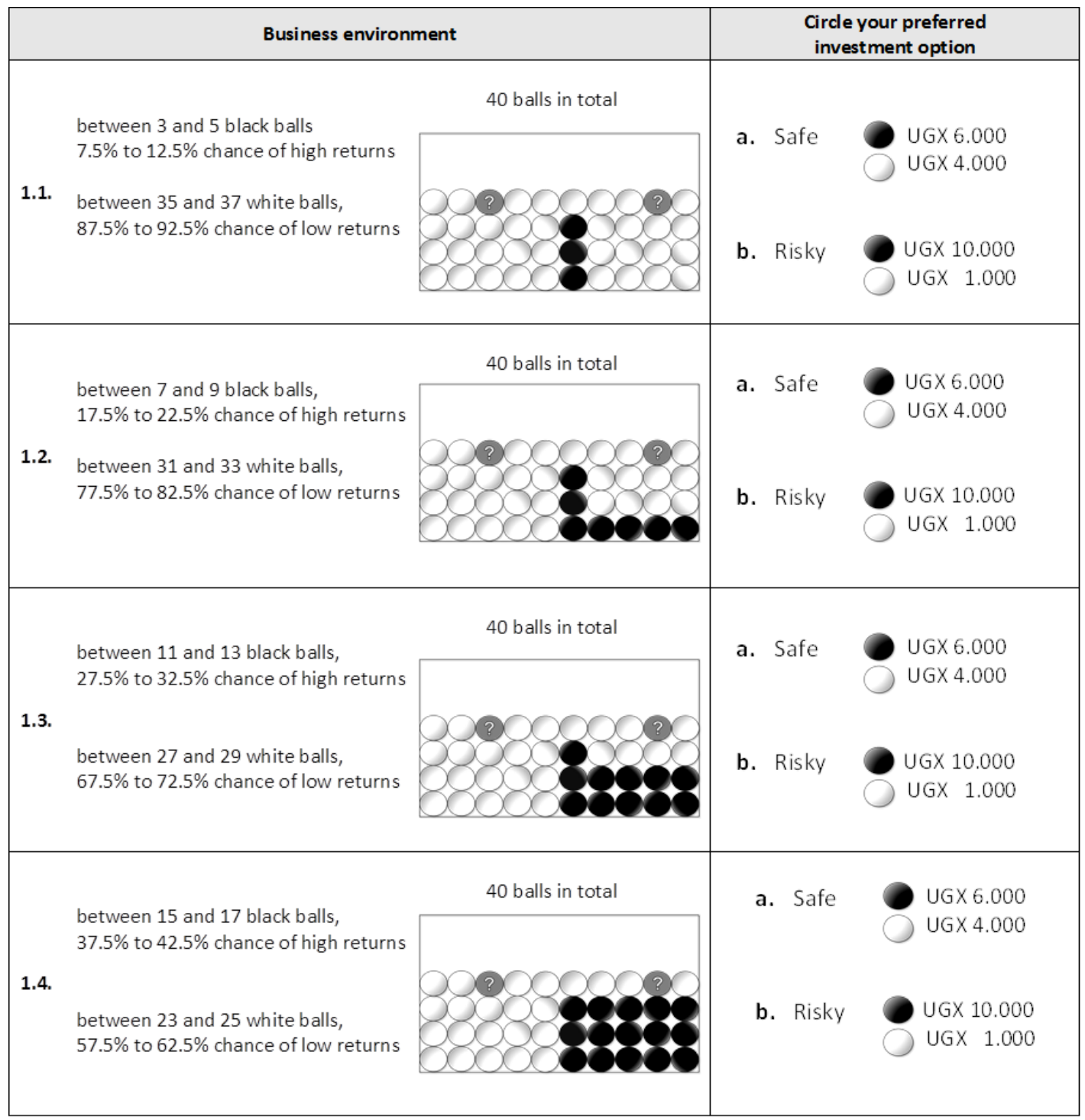


Figure 4: Decision sheet for the second experiment with low ambiguity (cont.)

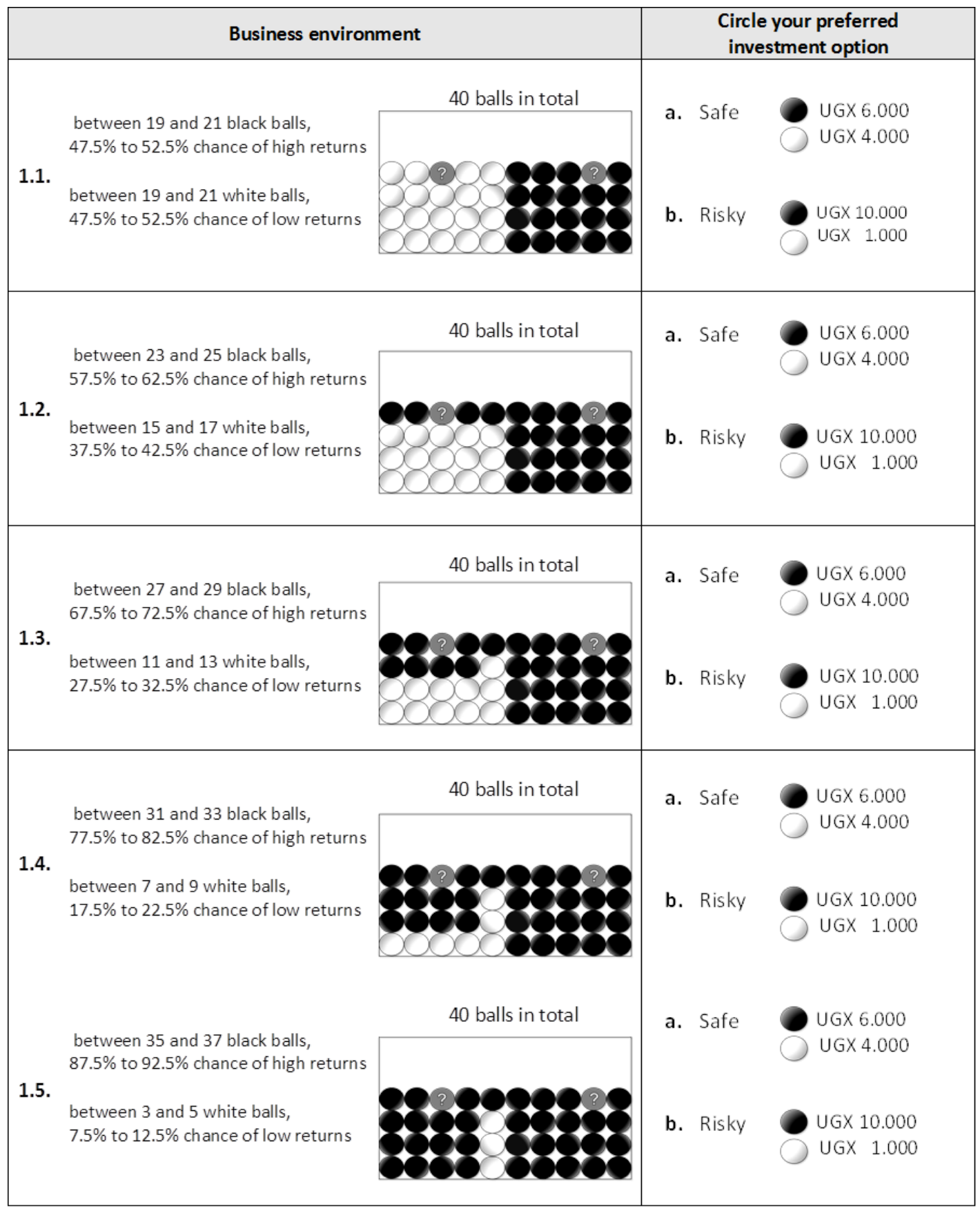


Figure 5: Decision sheet for the second experiment with medium ambiguity

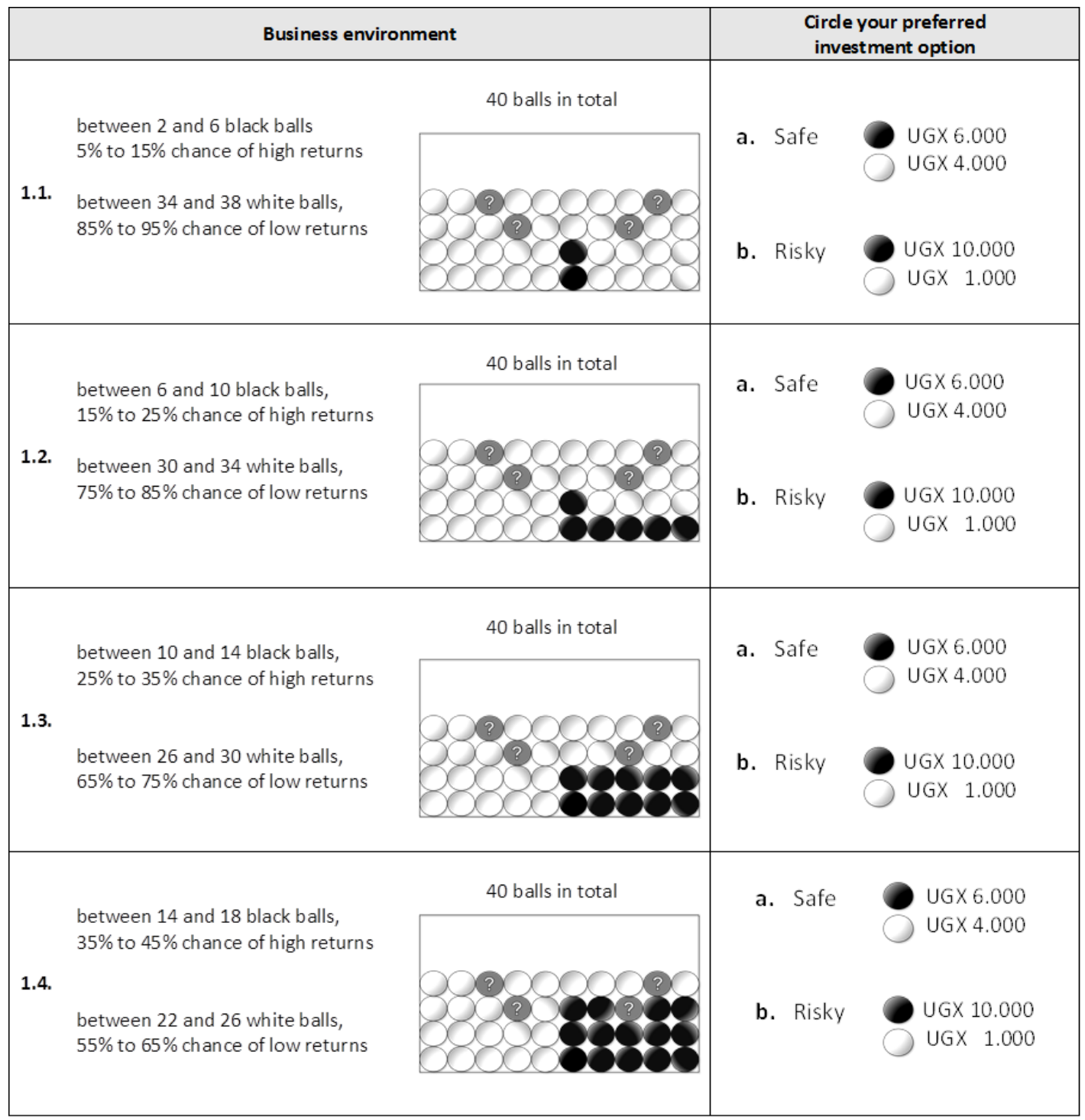


Figure 6: Decision sheet for the second experiment with medium ambiguity (cont.)

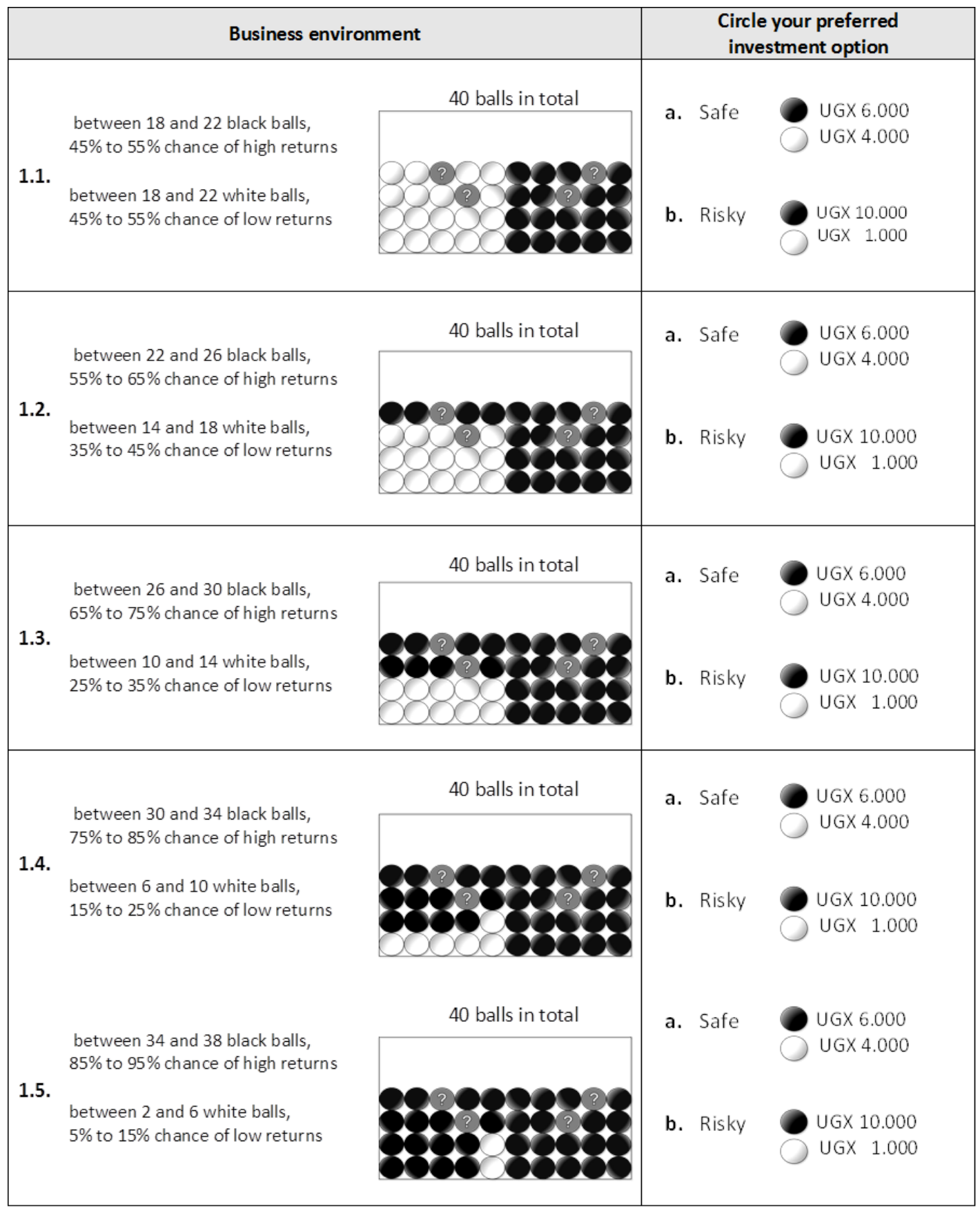


Figure 7: Decision sheet for the second experiment with high ambiguity

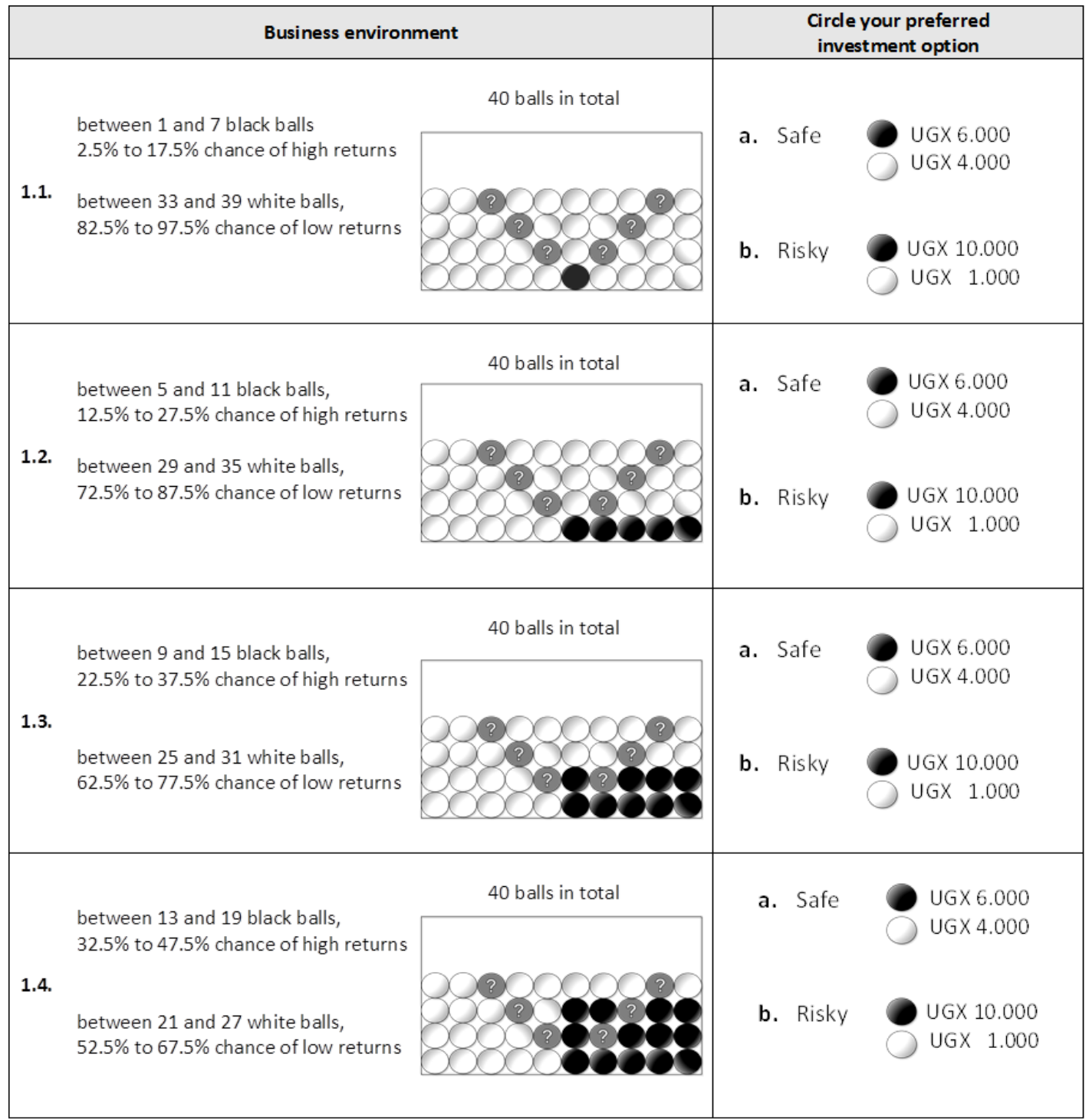


Figure 8: Decision sheet for the second experiment with high ambiguity (cont.)

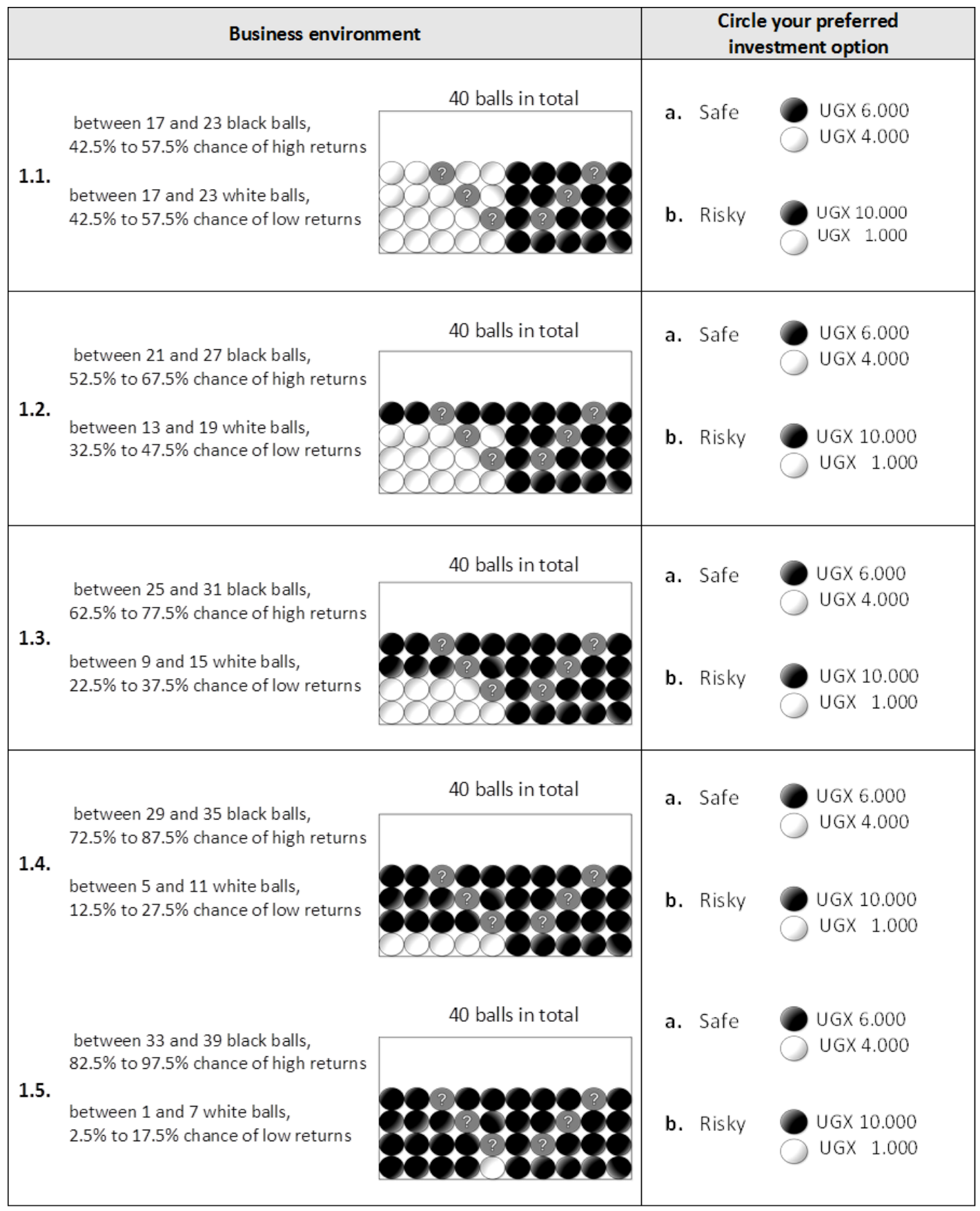

